\title{
Investigation of structural, electronic and optical properties of $\mathrm{KCdF}_{3}$
}

\author{
M. Nurullah Secuk, Emel Kilit Dogan, Murat Aycibin, Bahattin Erdinc, Harun Akkus*
}

Physics Department, Faculty of Science, Yuzuncu Yil University, 65080, Van, Turkey

\section{Email address:}

nurullahsechuk@gmail.com (M. N. Secuk), ekilit@yahoo.com (E. K. Dogan), murataycibin@yyu.edu.tr (M. Aycibin), bahattinerdinc@yyu.edu.tr(B. Erdinc),physicisthakkus@gmail.com (H. Akkus)

\section{To cite this article:}

M. Nurullah Secuk, Emel Kilit Dogan, Murat Aycibin, Bahattin Erdinc, Harun Akkus. Investigation of Structural, Electronic and Optical Properties of $\mathrm{KCdF}_{3}$, American Journal of Modern Physics. Vol. 2, No. 2, 2013, pp. 77-80. doi: 10.11648/j.ajmp.20130202.18

\begin{abstract}
The structural, electronic and optical properties of $\mathrm{KCdF}_{3}$ are investigated using the density functional theory (DFT) within the generalized gradient approximation (GGA). The calculated lattice parameters have been compared to experimental results and demonstrated to be in good agreement with them. The calculated electronic band structure of cubic $\mathrm{KCdF}_{3}$ shows that crystal has a indirect forbidden band gap with value of $2.95 \mathrm{eV}$ from the high symmetry point $\mathrm{R}$ to gamma point in the first Brillouin Zone (BZ). The optical spectra are investigated under the scissor approximation in the photon energy range, up to $30 \mathrm{eV}$. The dielectric function and some optical constants such as energy loss functions, reflectivity, extinction, and absorption coefficients, effective number of valance electrons and refractive index are calculated.
\end{abstract}

Keywords: Density Functional Theory, Electronic Structure, Optical Properties

\section{Introduction}

In recent years, extensive researches devoted to the experimental and theoretical study of structural phase transitions occurring in some fluoride compounds have let us to understand the physical properties of solids. Crystal structures of fluorides are prevalent in scientific and technological areas due to their electro-optic and electro-mechanic properties, nonlinearities, large dielectric constants, and fundamental interest of phase transitions. These structures undergo phase transitions as a result of rotations of the $F_{6}$ octahedral around one of the major crystallographic axes of the high temperature cubic symmetry phase. Therefore, the electronic structure and optical properties of fluorides are very important. The energy gap of these compounds lies in the ultraviolet region of the spectrum [1] that makes these crystals interesting for researchers. Among these important fluorides, $\mathrm{KCdF}_{3}$ crystal structure is particularly attractive due to luminescent property, laser medium and applications in radiation $[2,3]$. The $\mathrm{KCdF}_{3}$ crystal was found to have three temperatures of the phase transitions at 485,471 and $243 \mathrm{~K}$ [4]. The ideal structure of $\mathrm{KCdF}_{3}$ crystal compound has a simple cubic lattice where the potassium atom $(\mathrm{K})$ lies in the center of the cube, the cadmium atom $(\mathrm{Cd})$ lies at the corners of the cube and the fluorine atoms $(\mathrm{F})$ lie in the middle point of sides of the cube.
Moreover, $\mathrm{KCdF}_{3}$ crystal structure has been studied in detail. Huang et al. reported the preparation of $\mathrm{KCdF}_{3}$ nanocrystal [5]. Hidaka et al. presented the procedure of structural analysis of the phase III of $\mathrm{KCdF}_{3}$ containing six kinds of domains and discussed the results in terms of the successive condensation of soft phonon modes, using a full matrix least-squares method $[4,6]$. Hidaka et al. studied the successive structural transitions of $\mathrm{KCdF}_{3}$ by means of thermal diffusivity, polarized light scattering and X-ray diffraction [7]. Rousseau et al. considered the possible existence of phase transitions due to rotations of $\mathrm{CdF}_{6}$ octahedral in the $\mathrm{KCdF}_{3}$ compound using a simple model of force constants [8]. Darlington investigated the structures of two non-cubic phases of $\mathrm{KCdF}_{3}$ [9]. Chun-gang Duan et al. predicted that several fluoride $\mathrm{MCdF} 3(\mathrm{M}=\mathrm{K}, \mathrm{Rb}, \mathrm{Cs}$, TI) compounds could be formed in a ferroelectric crystal structure based on first-principle total energy calculations [1]. Kleemann et al. observed two weakly discontinuous structural phase transitions on single domains of $\mathrm{KCdF}_{3}$ in the temperature dependence of the linear birefringence [10]. Geguzina detected the nature and temperatures of the phase transitions of fluorides with the perovskite structure [11].

In our knowledge, no detailed first principle calculations of the physical properties of $\mathrm{KCdF}_{3}$ have been reported. In the present paper, the detailed calculations of the electronic and optical properties of $\mathrm{KCdF}_{3}$ are carried out using the 
pseudopotential method based on the DFT within GGA.

\section{Details of the Calculations}

$\mathrm{KCdF}_{3}$ has cubic structure at room temperature and belongs to the space group Pm-3m (No. 221). $\mathrm{KCdF}_{3}$ contains one molecule with five atoms per unit cell. The experimental lattice constant of $\mathrm{KCdF}_{3}$ crystal is $4.293 \AA$ [12]. At room temperature, $\mathrm{K}, \mathrm{Cd}$, and $\mathrm{F}$ ions are located at $(0.5,0.5,0.5)$, $(0.0,0.0,0.0)$, and $(0.5,0.0,0.0),(0.0,0.5,0.0),(0.0,0.0,0.5)$ in the unit cell of $\mathrm{KCdF}_{3}$, respectively.

The computer calculations were performed in the ABINIT code. After optimization process, the electronic and optical properties were calculated. The calculated results were obtained using GGA included the exchange-correlation effects [13] of DFT. The self-consistent norm-conserving pseudopotentials of FHI-type with a Troullier-Martins scheme [14] for all atoms of $\mathrm{KCdF}_{3}$ crystal were used. $4 \mathrm{~s}$ electron for $\mathrm{K}$ atom, $5 \mathrm{~s}$ and $4 \mathrm{~d}$ electrons for $\mathrm{Cd}$ atom, and $2 \mathrm{~s}$ and $2 \mathrm{p}$ electrons for $\mathrm{F}$ atom were considered as the true valence. The basis set for the electronic wave functions were chosen to be plane waves. The conjugate gradient minimization method [15] was used to solve Kohn-Sham equations [16] in the ABINIT code [17]. A good convergence for the total energy calculation with 50 Hartree cut off energy has been achieved using a $8 \times 8 \times 8$ Monkhorst-Pack mesh grid [18]. On the other hand, the irreducible BZ was sampled with $10 \mathrm{x} 10 \mathrm{x}$ 10 Monkhorst-Pack mesh grid to calculate the optical properties of $\mathrm{KCdF}_{3}$.

The electronic excitation spectrum of a material is usually defined in terms of frequency dependent complex dielectric function. The real and imaginary parts of the frequency dependent dielectric function contain all required respond information. Since optical properties of a solid are the response of the solid to electromagnetic perturbation occurring from incoming light to solid, the calculation of optical properties means computation of optical response function, namely dielectric function. If the imaginary component of dielectric function is known, the real part of the dielectric function can be obtained using the Kramers-Kronig relations. Rest of optical constants of the solid can be also calculated via imaginary dielectric function.

The Kohn-Sham equations determine the ground-state properties. Because the unoccupied conduction bands have no physical importance and a band gap problem appears at too low energy values, the self-energy effects must be included when the optical response calculations for semiconductors and insulators are made. In the GGA calculations, in order to include the self-energy effects, the scissor approximation [19] was used. In the approximation, we have shifted theoretical values of band gap by $4.5 \mathrm{eV}$ in order to match the experimental [20] and the theoretical band gap values.

\section{Result and Discussions}

In general, if total energy of a solid is known, one can determine the physical properties of the solid. As shown in Fig. 1, the theoretical lattice constant was calculated as $4.497 \AA$ by minimizing the total energy as a function of volume for the $\mathrm{KCdF}_{3}$ crystal. Comparing calculated lattice constant to experimental result [12], it is seen that they are in good agreement. For the rest of the calculations, the calculated lattice constant was used.

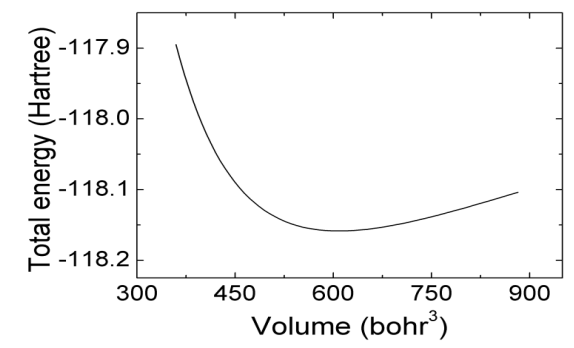

Figure 1. Dependence of total energy on unit cell volume for cubic $\mathrm{KCdF}_{3}$.

The investigation of energy band structure is very useful to be understood the electronic and optical properties of a material. Therefore, the calculated energy band structure of $\mathrm{KCdF}_{3}$ was obtained and demonstrated in Fig. 2, covering a wide range of energy from -21 to $12 \mathrm{eV}$, along high symmetry directions in the first BZ. The Fermi level is set as zero energy level and indicated by a horizontal dashed line in Fig. 2.

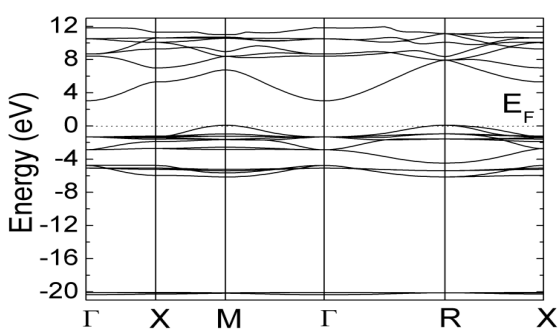

Figure 2. Calculated electronic band structure for cubic $\mathrm{KCdF}_{3}$.

The high symmetry points of irreducible BZ are marked with letters in Fig. 2. The maximum of valence band and the minimum of conduction band are located at the high symmetry points $\mathrm{R}$ and $\Gamma$, respectively, in the BZ. Therefore, the band structure for cubic $\mathrm{KCdF}_{3}$ shows indirect forbidden band gap from $\mathrm{R}$ to $\Gamma$.

The value of calculated indirect forbidden band gap is $2.95 \mathrm{eV}$. It is seen that the obtained band gap is less than the experimental band gap [20]. It is well known that the calculated band gap in DFT is always smaller than the experimental band gap. Probably, the underestimation of the band gap is due to the generic nature of the DFT. Thus, a scissors operator applied to the calculation of optical parameters is needed to shift all the conduction levels in order to agree with the measured value of the band gap.

Next, the optical properties of cubic $\mathrm{KCdF}_{3}$ were also studied. The optical properties can be obtained from the complex dielectric function which is usually connected with the electronic structure $[21,22]$. The frequency dependent imaginary part of complex dielectric function was calculated. Using the imaginary component of dielectric function, the 
real part of the dielectric function can be obtained by the Kramers-Kronig relations. The calculated optical functions neglecting all lattice vibrational effects and pertaining to only the electronic transitions were shown in Fig. 3. The optical response was chosen in the photon energy range of 0-30 eV considering the calculated band structure in Fig. 2. As a result of all the calculations, it is seen that the $0-30 \mathrm{eV}$ photon-energy range was sufficient for optical response of $\mathrm{KCdF}_{3}$.

The $\mathrm{KCdF}_{3}$ compound has a cubic structure which is

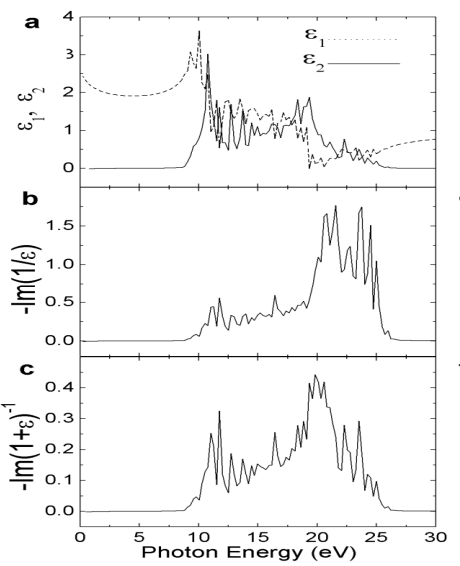

optically isotropic (anaxial) system. Therefore, the linear dielectric tensor for $\mathrm{KCdF}_{3}$ has one independent component. The calculated real and imaginary parts of the frequency dependent dielectric function were presented in Fig. 3. The peaks of the imaginary part of dielectric function in Fig. 3a correspond to the optical transitions from the valance band to the conduction band. As seen from the Fig. $3 \mathrm{a}$, the $\mathrm{KCdF}_{3}$ exhibits two fundamental oscillator bands at 9.50 and 10.74 $\mathrm{eV}$.

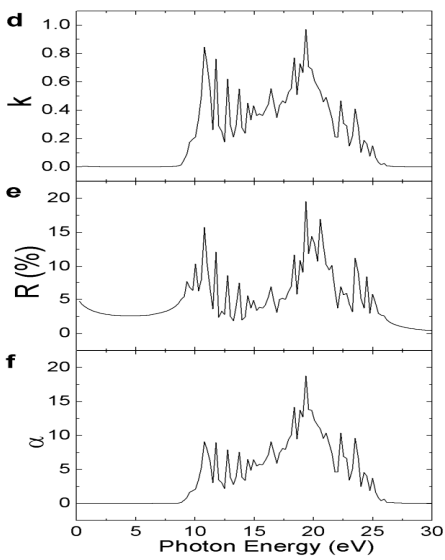

Figure 3. Calculated a) Real $\left(\varepsilon_{1}\right)$ and imaginary $\left(\varepsilon_{2}\right)$ parts of optical dielectric functions, b) energy loss function for volume, c) energy loss function for surface, d) extinction coefficient $(k)$, e) Reflectivity spectrum, e) absorption ( $\alpha$ ) coefficient for $K C d F_{3}$.

From the Fig. 3, while the $0-9.50 \mathrm{eV}$ photon-energy range is characterized by high transparency, no absorption and a small reflectivity which explains the origin of the peak structure in the reflectivity and absorption coefficient spectra, $9.50-10.75 \mathrm{eV}$ photon-energy range is characterized by strong absorption and appreciable reflectivity which means that optical absorption increases more quickly in this photon-energy range. Moreover, the photon-energy range of $10.75-25.00 \mathrm{eV}$ is characterized by high reflectivity. As can be seen from Fig. 3a, the static dielectric constant for $\mathrm{KCdF}_{3}$ was calculated as 2.44. The real part of dielectric function equals to zero at 19.31 and $19.43 \mathrm{eV}$.

The calculated energy-loss functions of volume and surface for $\mathrm{KCdF}_{3}$ were illustrated in Figs. $3 \mathrm{~b}$ and $3 \mathrm{c}$, respectively. The volume loss function gives a description about the energy loss of the fast electrons passing through the material. One can see from these figures, the energy-loss functions for volume and surface shows mainly many intense maximum peaks. The sharp maxima in the volume-loss function are associated with the excitation of plasma. The energy values corresponding to peaks of volume loss function coincide with the energy values corresponding to zero values of real part of dielectric function.

Moreover, the extinction coefficient, reflectivity spectrum, and absorption coefficient are shown in Figs. 3d, 3e and 3f, respectively.

The sum rule can be used to determine the effective number of valence electrons contributing to optical transitions [21, 22]. An estimate of distribution of oscillator strengths can be obtained for both intraband and interband transitions. As can be seen from Fig. 4a, the effective number of valance electrons is zero up to photon energy values of $9.5 \mathrm{eV}$, then rises rapidly and reaches a saturation value at about $25.00 \mathrm{eV}$. This shows that the interband transitions do not include deep-lying valence states.

The calculated refractive index was shown in Fig. $4 \mathrm{~b}$. For the calculation of the refractive index, $0-3 \mu \mathrm{m}$ photon-wavelength range was taken and it was sufficient enough for the calculations. This figure represents spectral dependence of the calculated main refractive index for cubic $\mathrm{KCdF}_{3}$ in a wide wavelength range and reveals the decreasing behavior of refractive index $(n)$ with the transition from the intrinsic absorption region towards long wavelengths where a normal dispersion takes place. Using $n=n$ ( $\lambda$ ) dependence, the maxima were obtained at $\lambda=0.12 \mu \mathrm{m}$ with the values of $n$ as 1.92 .
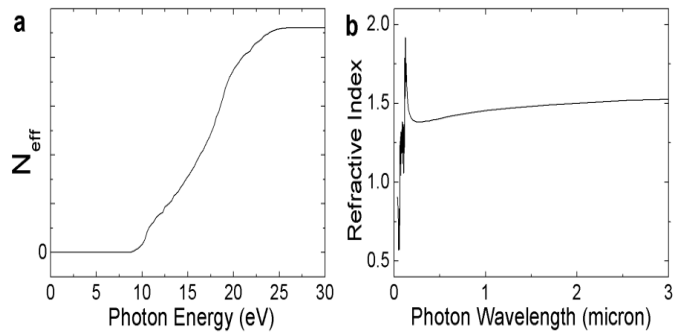

Figure 4. a) Effective number of valance electrons vs photon energy b) Refractive index vs photon wavelength for $\mathrm{KCdF}_{3}$.

\section{Conclusions}

The aim of the present work is to investigate electronic 
and optical properties of cubic $\mathrm{KCdF}_{3}$ compound using the DFT under the GGA. As a result of calculations, it was found that the forbidden band gap of cubic $\mathrm{KCdF}_{3}$ is indirect character in the symmetry direction from $\mathrm{R}$ to $\Gamma$ in the $\mathrm{BZ}$. The calculated value of forbidden band gap is $2.95 \mathrm{eV}$ and is smaller than its experimental value due to the generic nature of the DFT. The photon energy dependent dielectric functions as well as related quantities such as energy-loss functions for volume and surface, extinction and absorption coefficients, reflectivity and refractive index were investigated. Moreover, the effective number of valence electrons per unit cell participating in the interband transitions was studied. The results of implemented structural optimization by using the GGA are in excellent agreement with the available experimental data. For optical and electronic properties, the calculated results cannot be compared with any data due to the lack of any experimental or theoretical work previously performed.

\section{Acknowledgements}

This work has been supported by The Unit of Scientific Research Projects of Yuzuncu Yil University under project No. 2011-FED-B010.

\section{References}

[1] C.-g. Duan, W. N. Mei, J. Liu, W.-G. Yin, J. R. Hardy, R. W. Smith, M. J. Mehl, and L. L. Boyer, "Electronic properties of NaCdF3: A first-principles prediction," Phys. Rev. B, vol. 69 (2004) pp. 033102-1- 033102-4.

[2] A. Gektin, I. Krasovitskaya, and N. Shiran, "High-temperature thermoluminescence of $\mathrm{KMgF} 3$-based crystals," Journal of Luminescence, vol. 72-74 (1997) pp. 664-666.

[3] M. Mortier, I. Gesland, and M. Rousseau, "Experimental and theoretical study of second-order Raman scattering in BaLiF3," Solid State Commun., vol. 89 (1994) pp. 369-371.

[4] M. Hidaka, S. Hosogi,, M. Ono and K. Horai, "Structural phase transitions in $\mathrm{KCdF}_{3}$," Solid State Commun., vol. 23 (1977) pp. 503-506.

[5] B. Huang, J. M. Hong, X. T. Chen, Z. You, and X. Z. You, "Mild solvothermal synthesis of $\mathrm{KZnF}_{3}$ and $\mathrm{KCdF}_{3}$ nanocrystals," Materials Letters, vol. 59 (2005) pp. 430-433.

[6] M. Hidaka, and S. Hosogi, "The crystal structure of $\mathrm{KCdF}_{3}$," J. Phys. France, vol. 43 (1982) pp. 1227-1232.

[7] M. Hidaka, Z. Y. Zhou, and S. Yamashita, "Structural phase transitions in $\mathrm{KCdF}_{3}$ and $\mathrm{K}_{0.5} \mathrm{Rb}_{0.5} \mathrm{CdF}_{3}$," A Multinational Journal, vol. 20 (1990) pp. 83-94.

[8] M. Rousseau, J. Y. Gesland, J. Julliard, J. Nouet, J. Zarembowitch, and A. Zarembowitch, "Crystallographic, elastic, and Raman scattering investigations of structural phase transitions in $\mathrm{RbCdF}_{3}$ and $\mathrm{TlCdF}_{3}$," Phys. Rev. B, vol. 12 (1975) pp. 1579-1590.

[9] C. N. W. Darlington, "Phase transitions in $\mathrm{KCdF}_{3}$," J. Phys. C: Solid State Phys., vol. 17 (1984) pp. 2859-2868.

[10] W. Kleemann, J. Y. Gesland, and J. Nouet, "Linear birefringence studies of structural phase transitions of $\mathrm{KCdF}_{3}$," Solid State Communications, vol. 26 (1978) pp. 583-588.

[11] G. A. Geguzina, "Interatomic Bond Strains-Phase Transition Temperatures Correlations for Perovskite Compounds," Integrated Ferroelectrics, vol. 64 (2004) pp. 61-68.

[12] R. L. Moreira, and A. Dias, " Comment on prediction of lattice constant in cubic perovskites," J. Phys. Chem. Solids, vol. 68 (2007) p. 1617-1622.

[13] J. P. Perdew, K. Burke and M. Ernzerhof, "Generalized Gradient Approximation Made Simple," Phys. Rev. Lett., vol. 77 (1996) pp. 3865-3868.

[14] N. Troullier and J. L. Martins, "Efficient Pseudopotentials for Plane-Wave Calculations.," Phys. Rev. B, vol. 43 (1991) pp. 1993-2006.

[15] M. C. Payne, M. P. Teter, D. C. Allan, T. A. Arias and J. D. Joannopoulos, "Iterative minimization techniques for ab initio total energy calculations: molecular dynamics and conjugate gradients,” Rev. Mod. Phys., vol. 64 (1992) pp. 1045-1097.

[16] W. Kohn and L. J. Sham, "Self-Consistent Equations Including Exchange and Correlation Effects," Phys. Rev., vol. 140 (1965) pp. A1133- A1138.

[17] X. Gonze, J. M. Beuken, R. Caracas, F. Detraux, M. Fuchs, G. M. Rignanese, L. Sindic, M. Verstrate, G. Zerah, F. Jollet, M. Torrent, A. Roy, M. Mikami, P. Ghosez, J. Y. Raty, D. C. Allan, "First-principle computation of material properties: the ABINIT software project," Computational Materials Science, vol. 25 (2002) pp. 478-492.

[18] H. J. Monkhorst and J. D. Pack, "Special points for Brillouin-zone integrations," Phys. Rev. B, vol. 13 (1976) pp. 5188-5192.

[19] J. L. P. Hughes and J. E. Sipe, "Calculation of second-order optical response in semiconductors," Phys. Rev. B, vol. 53 (1996) pp. 10751-10763.

[20] Y. Chornodolskyy, S. Syrotyuk, G. Stryganyuk, A. Voloshinovskii, and $\mathrm{P}$. Rodnyi, "Electronic energy structure and core -valance luminescence of $\mathrm{ABX} 3(\mathrm{~A}=\mathrm{K}, \mathrm{Rb}, \mathrm{Cs} ; \mathrm{B}=\mathrm{Ca} ; \mathrm{X}=\mathrm{F})$ crystals," Journal of Physical Studies, vol. 4 (2007) pp. 421-426.

[21] M. Fox, Optical Properties of Solids. Oxford University Press, Oxford, UK, 2002, pp. 2-7.

[22] H. R. Philipp, and H. Ehrenreich, "Optical Properties of Semiconductors," Phys. Rev., vol. 129 (1963) pp. $1550-1560$. 